\title{
DISTRIBUTION OF LIGHT: OUTER REGIONS
}

\author{
Massimo Capaccioli \\ Institute of Astronomy \\ University of Padova \\ 35122 Padova \\ Italy
}

\section{INTRODUCTION}

The data base of photometric properties of galaxies has grown enormously in the last few years. Surveys in various colors, detailed studies of selected objects, and a swarm of papers with underlying photometric information have invaded the market, fostered by advances in the technology of observations and by improvements and wider availability of reduction facilities. A bibliographical compilation, of great use in the jungle of old and modern references, has been published by Davoust and Pence (1982), and is currently updated (Pence and Davoust 1985).

The preference given by observers to early-type galaxies is in line with the revival of interest for ellipticals, triggered, about 10 years ago, by unexpected kinematical findings (Bertola and Capaccioli 1975, Illingworth 1977). Indeed, photometry has proven a source of original new input to the growing picture on E galaxies; the discoveries of isophotal twisting (whose paternity, although controversial, should be fairly attributed to Williams and Schwarzschild, 1979) and of shells (Malin and Carter 1980), are just two examples. The photometric technique has always been a fundamental complement to other lines of investigation; for instance, lacking better information, light profiles are used to describe the projected distribution of matter under the assumption that $\mathrm{M} / \mathrm{L}$ is constant with radius. Furthermore, after Oemler (1976), surface photometry of early-type galaxies has become a standard tool to investigate tidal interactions, merging and cannibalism. Elliptical and lenticular galaxies are expected to share more homogeneous properties than later types, in absence of external disturbances; they also occur at different levels of clustering. Thus their colors, structure and outer light profiles are all probes of induced modifications and their relation to environmental conditions.

Many modern surveys are based upon CCD data (e.g., Malumuth and Kirshner 1984, Kent 1984, Lauer 1985, Djorgowski 1986). Unfortunately, however, the area covered by presently available detectors (a few arc-minutes ${ }^{2}$ at conventional focal-plane scales) is insufficient to encompass large galaxies, which are the most convenient photometric targets (because of better resolution, higher integrated S/N at each isophote, reduced effects of scattered light and local crowding). One consequence is that, so far, our best machinery for surface photometry has contributed primarily to the knowledge of the brighter regions of galaxies. Most, if not all of the modern investigations extending to limiting surface brightness levels $\left(\sim 10^{-2}\right.$ 
of the night sky luminosity or fainter), are still based 1) on photographic material collected with large field telescopes, now digitized and reduced with improved techniques, and 2) on photoelectric observations (aperture photometry and drift scans). A remarkable exception concerns the study of peculiar outer structures with CCD data (e.g., Fort et al. 1986).

Before starting this review, it seems worthwhile to comment on the word 'outer' appearing in the title. Cores and outskirts of galaxies pose different problems of both technical and astrophysical nature, justifying the operative separation of light profiles into an inner and an outer region. Still, we should remember that the photometric analysis must be always carried out over the complete luminosity range, since it is often the case that the properties of the outer profile are determined relative to those of the inner ones, and vice-versa.

In the following discussion, much space is given to selected technical aspects of the game. Their complexity explains the small enthusiasm for and the skepticism about surface photometry at faint levels, and also accounts for the poor quality of some of the results. In addition to a review of the data and their interpretations, empirical fitting formulae, particularly the $r^{1 / 4}$ law (de Vaucouleurs 1948), are discussed for the benefit of theorists, commenting on their deviations from observations at large and small scales. Finally, we will speculate about misclassifications and contamination of the elliptical class by lenticulars. Dwarf E galaxies are deliberately ignored, since they appear to form a family which is physically distinct from that of ordinary ellipticals (Kormendy 1985, Ichikawa et al. 1986; see the contribution by Nieto in this volume). Previous reviews dealing with this subject were published by de Vaucouleurs $(1959,1979,1983)$, Kormendy $(1980,1982)$, and Capaccioli (1984a).

\section{TECHNICAL PROBLEMS AND SOURCES OF ERRORS}

The following outline of the technical problems in faint surface photometry is not specific to elliptical galaxies. It is partly based on a paper by Capaccioli and de Vaucouleurs (1983; hereafter CdV), which can be consulted for quantitative information. Similar discussions are scattered in the literature.

\subsection{Calibration}

Individual pixels of CCD's are linear over a wide range, and sensitivity variations across the detector can be readily corrected. Photographic emulsions, on the contrary, are not linear. This fact does not prevent an accurate intensity calibration, provided that the material is carefully processed and digitized, and that the external sensitometer is properly designed (Fig. 1). Very little can be added to the extensive discussion by de Vaucouleurs (1983). Still, we want to reiterate the complaint about several telescopes whose superb photographic material is degraded by inadequate sensitometry (particularly noteworthy is the light leakage from the densest spots occurring in most of the Kitt Peak type projectors). In any case, calibration errors weight less at faint than bright light levels (the H-D curve is linear at the sky density in well exposed plates). This tolerance justifies the use of an internal calibration whenever strictly needed, e.g. for archival plates, although the recovered linearization curve can never extend below the sky density (see Piotto 1986, for an interesting new method of internal calibration, well suited for galaxy photometry). In closing, it is important to emphasize that calibration is not the limiting factor in photographic surface photometry. 


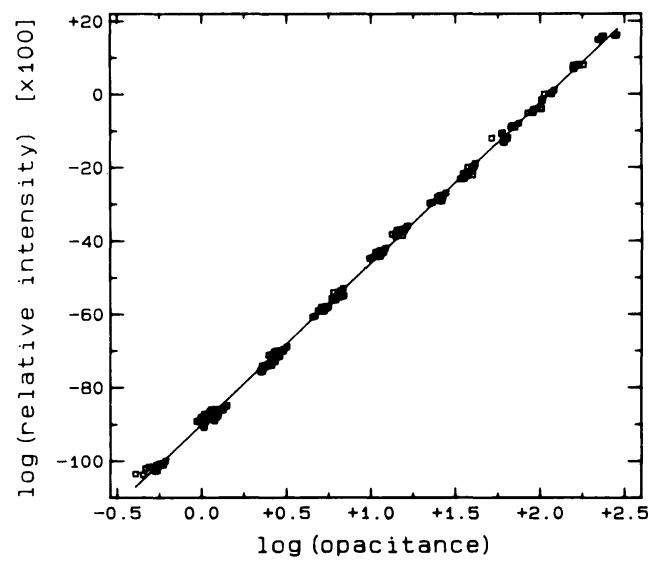

Figure 1. Plot of nominal intensity vs. opacitance (de Vaucouleurs 1968) for the individual calibration spots of 6 unbaked IIa-O plates exposed at the ESO Schmidt telescope through a GG385 filter. The mean linear relation has a slope of 0.438 with an uncertainty of $\pm 0.3 \%$ (Capaccioli and Rampazzo 1986).

\subsection{Background removal and related problems}

As the distance from center increases, the surface brightness of a galaxy fades smoothly until it merges into the diffuse light of the night sky. Thus, the most critical step in faint surface photometry is the removal of a noisy, low spatial frequency component, which is hundreds of times stronger than the outskirts of the superimposed object. An error in the adopted sky level may have dramatic consequences on the apparent luminosity profiles. For instance, depending on the sign of the systematic error, a pure $r^{1 / 4}$ profile will mimic a tidal extension or a cutoff ( $c f$. Fig. 1 in $\mathrm{CdV}$ ). The background problem, common to all observational techniques, increases significantly whenever the blank sky cannot be recorded simultaneously with the galaxy, since the luminosity of the night glow is not constant (random variations up to $10 \%$ may occur in a few minutes; de Vaucouleurs 1958). This is the typical difficulty faced in CCD photometry of galaxies with large angular sizes. Blank sky exposures preceding and following the galaxy exposure, hated by observers because they are time consuming, may help to beat the background fluctuations. We will not comment here on other 'tricks' adopted by CCD observers to recover the sky level from a frame completely filled by a galaxy image (for instance, the tautology of seeking it by the assumption that galaxy profile follows a given photometric law).

Since the beginning of galaxy photometry (Hubble 1930), the low frequency component of the local background is estimated by some kind of interpolation of the signal from the blank sky areas surrounding the galaxy image. Computer procedures, inspired by the 2-D mapping pioneered by Jones et al. (1967), are now currently in use. It would be difficult to list all the various versions and recipes (see, e.g., the median filter approach by Sulentic and Lorre, 1983). In the Interactive Numerical Mapping Package, a dedicated procedure developed at the Padova Observatory to reduce PDS scans of plates (Barbon et al. 1976a,b), the background contribution is modelled by fitting a canonical 2-D polynomial to the density readings in an 'outer field' encircling the galaxy. Large images, such as bright stars, are masked interactively, while the high frequency components (small stars, emulsion defects, dust, etc.) are taken out by clipping the histogram of the $(O-C)$ residuals. The fitting-rejection procedure is repeated until the standard deviation $\sigma$ and the skewness of the $(O-C)$ histogram converge. At each cycle, the low- 
est possible order (from 1 to 5 ) is chosen for the polynomial on the basis of some graphical tests. The final $\sigma$ resulting from reduction of limiting Schmidt exposures on unbaked IIa-O plates is of the order of a few 0.001 density units (Capaccioli and Rampazzo 1986); larger values would not permit us to attain the threshold surface brightness $\mu=28-29 B-s s$ (abbreviation for $B-m a g / \operatorname{arcsec}^{2}$ ). Often a first order polynomial is inadequate to map the complicated pattern of the 'outer field'. Since systematic deviations from linearity may exist (e.g., vignetting in prime focus plates), it is unsafe to prejudicially exclude fitting surfaces other than a plane (cf. Schombert 1986).

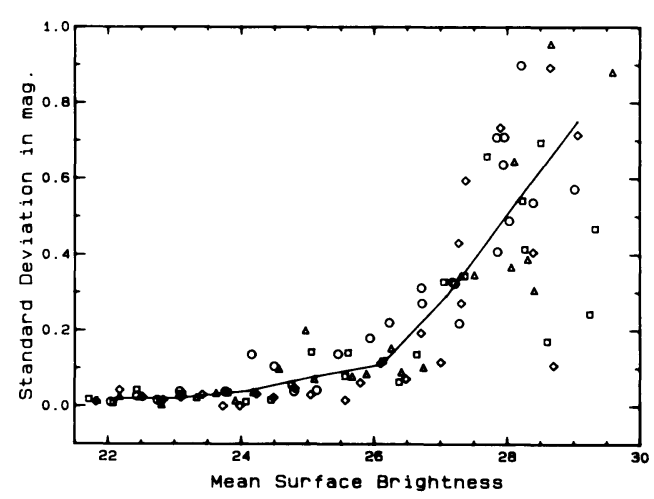

Figure 2. Internal (random) errors vs. surface brightness for the main axis light profiles of the S0 galaxy NGC 3115 from two pairs of deep ESO and UK Schmidt plates (Capaccioli et al. 1986a). The mean trend (solid line) shows that, at $\mu \simeq 26 B$-ss, the uncertainty of the photometric measurements begins to increase rapidly, reaching $100 \%$ at $\mu \simeq 28.5 B$-ss, in agreement with the threshold estimated by $\mathrm{CdV}$. Consistent values (for the total errors in the V-band) are reported by Schombert (1986).

After several years of use and continuous testing by numerical simulations, the INMP has proven quite effective for removing the background in the absence of complicated situations (see Fig. 2). There are cases, however, in which systematic errors are difficult to control and to evaluate. They occur, for instance, in dense cluster cores, where the outskirts of galaxies overlap and the background becomes a matter of definition, or when a galaxy has close companions, or it is embedded in the corona of a bright star (e.g., NGC 404; Barbon et al. 1982) or wrapped within a high-latitude reflection nebulosity ( $c f$. CdV). In addition, many methodological issues remain unsolved. They are related to the complexity of the sources contributing to the blank sky pattern. For example, low frequency sensitivity variations across a plate and vignetting are different phenomena which, in principle, should be treated separately. Efforts in this direction are rare, and possibly unjustified by the magnitude of the residual sources of systematic and accidental errors. One is the statistical fluctuation in the number density of sub-threshold stars and galaxies, first noted by Miller (1963) and investigated by de Vaucouleurs and Capaccioli (1979; hereafter dVC) in connection with NGC 3379. Unavoidable from both ground and space, this cosmic noise sets at $\mu \simeq 30 B-s s$ the impassable lower threshold for photometric measurements.

\subsection{Scattered light}

In addition to classical seeing convolution affecting high frequency structure, the light of a galaxy image is also re-distributed by telescopic, atmospheric and Rayleigh scattering (acting at different scales; see Fig. 4 in CdV). It is mostly the energy of the brighter parts which is shed into the external regions. In their study of NGC 
$3379, \mathrm{CdV}$ show that the outer $\left(r>30^{\prime \prime}\right)$ radial profile of the scattered light is approximately described by the complete Point Spread Function scaled to the same effective magnitude as the galaxy. With this simple model, it is easily verified that the contamination of the galaxy profile by scattered light increases systematically with radius until the latter becomes the dominant signal; the breakpoint occurs at a surface brightness whose value $\mu_{b}$ depends on the photometric law in the object and its scaling factor. For the model of NGC $3379\left(r_{e}=56^{\prime \prime}\right), \mu_{b}=32 B-$ $s s$, and the excess light is less than $0.14 \mathrm{mag}$ at all $\mu<28 B$-ss (CdV). The relation, $\mu_{b}=\mu_{e}+11.44-1.52 \log r_{e}$, holds for an $r^{1 / 4}$ circular galaxy in the range $30 "<r_{e}<100 "$.

\subsection{Light profiles: which and how many?}

A single intensity profile at a given position angle, coupled with the geometrical information (ellipticity and orientation profiles), is often thought sufficient for modelling the 2-D light distribution of an elliptical galaxy. This presumption, together with the need for increasing the significance of measurements at faint levels, has made popular the use of the equivalent profile, in which the surface brightness is related to the radius $r^{*}$ of the corresponding circularized isophote; for purely elliptical isophotes, $r^{*}=\sqrt{a b}$. Profiles derived by the ellipse fitting technique fall in this category. Isophotes of real galaxies, however, are often not pure ellipses (Jedrzejewski, this volume), and equivalent profiles (or other mean profiles, as in King 1978), may obscure structural details and encourage incorrect conclusions (see Fig. 3 and section 5 ). In other words, experience suggests that, even for apparently bona fide elliptical galaxies, it is always desirable to produce at least two distinct and unsmoothed luminosity profiles along the main axes.

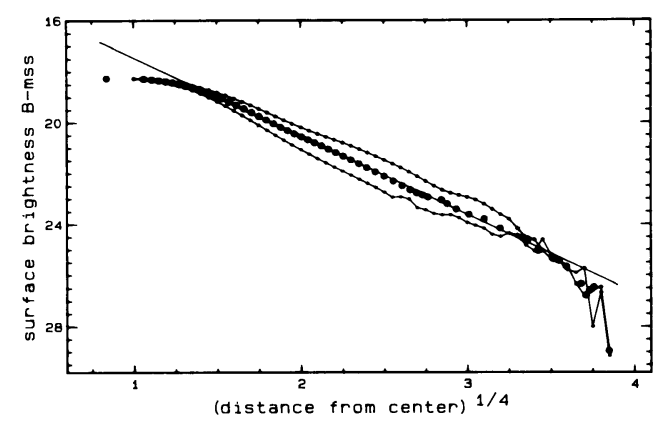

Figure 3. Major and minor axis intensity profiles (small dots) of NGC 4125 from two low resolution plates of the Asiago 1.22-m reflector. The galaxy, classified E4 in RC2, is actually a lenticular with peculiar kinematics (Bertola et al. 1982). Large dots trace the equivalent profile, which, contrary to the profiles along the main axes, is well represented by an $r^{1 / 4}$ law (solid line) down to $\mu \simeq 26 B$-ss.

At very faint levels, especially when statistical fluctuations cause the occurrence of negative intensity values, resolution must be sacrificed in order to reduce the noise. An efficient method of extracting smooth profiles from clean 2-D maps is to sample them with a variable aperture in such a way that $S / N$ remains approximately constant (Capaccioli and Rampazzo 1986). For an $r^{1 / 4}$ galaxy, a simple rule is to center the aperture along one axis at distances equally spaced in $r^{1 / 4}$ units, and choose the radius equal to half of the spacing. Anomalous values are easily sorted out by checking the agreement between the geometrical center of the aperture and the light baricenter (no difference should occur at large radial distances). This technique, coupled with an efficient algorithm for removing the stars (e.g., 
the package 'DAOPHOT' developed for stellar photometry; Stetson 1983), allows a gain of $\sim 0.5 \mathrm{mag}$ over conventional procedures.

\subsection{Photometric zero point calibration}

In photographic work the zero point of the photometric scale is usually set by a comparison with photoelectric measurements. In most cases only photoelectric integrated magnitudes within centered apertures are available. The comparison is straightforward, provided that the photographic photometry has no systematic scale error, especially near the galaxy center, where the luminosity gradient is steep. This is not the case for the limiting exposures used to map the faint outskirts of galaxies, due to saturation (e.g., deep Schmidt plates are not reliable for $\mu<22 B$ ss). Therefore, the outer profiles of galaxies can only be connected by matching to photoelectrically calibrated inner profiles, if available. This procedure, needed in any case to produce the complete light distribution from different exposures, may propagate systematic errors and modify the trend of the final luminosity profile. Often the problem is complicated by the use of heterogeneous material, e.g. plates and CCD frames, without proper allowance for the color equations of the various devices. The quality of the result from this pipeline process cannot easily be controlled with photoelectric photometry, 1) because the data at large apertures is scarce, often old, and unreliable, and 2) since integration tends to absorb even large errors in the luminosity gradients, transferring them into a shift of the photometric zero point.

\subsection{Ultimate limit and photometric standards}

Taking into account all sources of errors, CdV estimate that, while it is possible to make detections, it is difficult, if not impossible, to obtain reliable quantitative information at brightness levels fainter than $\mu \simeq 28.5 B-s s$. Due to the nature of some sources of errors, the limit is unlikly to improve significantly by future technical advances. The solid line in Fig. 2 is indicative of the expected uncertainty in the best possible situation, provided that all systematic errors are removed.

Facing the problem of photometric accuracy, in 1962 the Working Group on Galaxy Photometry of IAU Commission 28 (IAU Trans., XIB, p. 304) stressed the need for standards and proposed a short list of candidates for the various morphological types. However, not much effort has been put into this important project by the community, and so far only three early-type galaxies have been studied in detail, combining a large set of old and new data, namely NGC 3379 (dVC, Nieto 1983a,b, Capaccioli et al. 1986b), M87 (de Vaucouleurs and Nieto 1978, 1979) and NGC 3115 (Capaccioli et al. 1986a). Several observers have already used this precise information as a reference to evaluate the accuracy of their work. They have simultaneously created a new body of data to be incorporated in a forthcoming revision of the standards themselves.

\section{STATE OF THE ART}

In a classical paper King (1978) gave light profiles and geometrical parameters for 17 early-type galaxies; the limiting surface brightness was $\mu \simeq 28 B$-ss and, outside cores, the photometric accuracy was usually better than $15 \%$ at all $\mu<$ $26 B$-ss (Capaccioli 1984a). With few exceptions, previously published results are 
affected by large errors. Figure 5 in Capaccioli (1984b) illustrates the situation for Fraser (1977) and Liller $(1960,1966)$. The comparison with unpublished data from Benacchio and collaborators, discloses a systematic trend of the magnitude differences $v s$. surface brightness which, as already noted by Burstein (1979), is particularly large for Liller (up to $1 \mathrm{mag}$ ). The scenario does not improve even for selected well known galaxies (see the case of M87 analysed by Carter and Dixon, 1978). In this situation most of the statements about the outer light profiles of ellipticals made prior to King's (1978) study should be taken with reservations.

Modern studies tend to be more reliable than older ones. Their quality is often clearly established by comparison with a standard galaxy (e.g., Kent 1984, Michard 1985). There are large surveys conceived for statistical purposes, which have a declared limited precision in the individual values (Binggeli et al. 1984). Other surveys which are not very deep (e.g., Watanabe 1983, and CCD surveys), and others which claim an accuracy of 0.9 at $\mu=29 V-s s$ (Schombert 1986). The huge project carried out by Lauberts and Valentijn on the ESO Survey plates deserves special mention. It will make available, in a few years, a deep and fairly precise photometric sample for a total of 16,000 galaxies of all types, including 2,500 early-type systems (Lauberts and Valentijn 1984). The flood of data will be filtered and summarized in the forthcoming third edition of the 'Reference Catalogue of Bright Galaxies' by de Vaucouleurs and collaborators.

However, even for the modern data, errors lie in wait, as can be illustrated by the two following examples.

i. NGC $4406=$ M86, a well known early-type giant at the end of the Markarian chain in the Virgo cluster, is a classical target for surface photometry ( $c f$. the list of references in Davoust and Pence, 1982, 1985). Its remarkable properties have been compiled by Capaccioli et al. (1983). When plotted together, the light profiles from the various sources exhibit a very large scatter at all $\mu>24 B$-ss. The explanation for this unusually strong disagreement is in the huge halo surrounding M86, which is almost as large as that of M87 (Capaccioli, 1984a). Michard (1985) is the only study where this halo has been taken into account while determining the background level. Uncorrected, the halo results in a spurious increase of the gradient in the outer light profile, with amplitude depending on the individual choice of the fictitious blank sky.

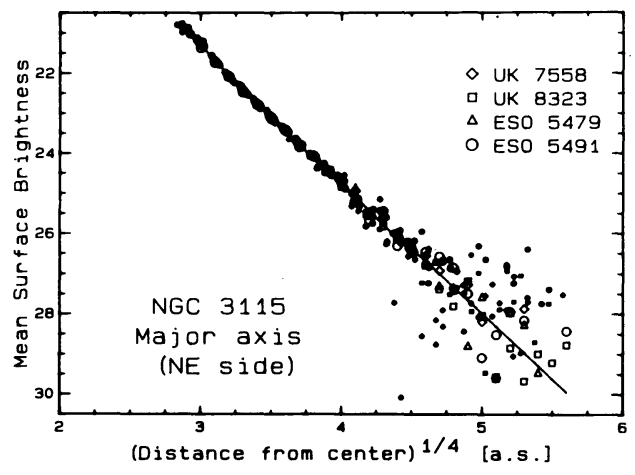

Figure 4. N-E side of the luminosity profile of NGC 3115 from the same four Schmidt plates of Fig. 2, showing the consistency of this material to the limit of detection. Small symbols represent unsmoothed cross-sections, large symbols are measurements with a software defined aperture with variable radius ( $c f$. section 2.4). Negative fluctuations were accounted for in computing the mean (solid) line.

ii. NGC 3115 is the prototype of S0 galaxies and one of the standards proposed by the Working Group on Galaxy Photometry. Using a large collection of plate ma- 
terial from various telescopes and a well established methodology, Tsikoudi (1978, 1979) obtained the main axis light profiles extending to $\mu=28 B-s s$, from which she derived important conclusions about fitting laws and the presence of photometric components. More recently, Capaccioli et al. (1986a) have repeated the work, using four deep plates, taken with the ESO and UK Schmidt telescopes, to record the faint outer regions. From the internal agreement (Fig. 4) and the external comparison with the photoelectric measurements by Miller and Prendergast (1968), and from an independent study of NGC 3379 done with similar Schmidt material, these authors estimate an accuracy better than $0.2 \mathrm{mag}$ for $\mu<26 \mathrm{~B}-\mathrm{ss}$ (see also Capaccioli et al. 1984a). The main axis light profiles of NGC 3115 from the two sources are plotted in Fig. 5; the inner part in Capaccioli et al. comes from the reduction of four CFH plates. Besides a zero point difference of about $0.5 \mathrm{mag}$, removed in the figure by a vertical shift of Tsikoudi's data, there is a disagreement between the two sources, starting at $\mu \simeq 24 B$-ss on both axes and increasing outwards to a maximum of $2 \mathrm{mag}$ at the largest radial distance. We face again a problem in the background level, affecting at least one of the two studies.

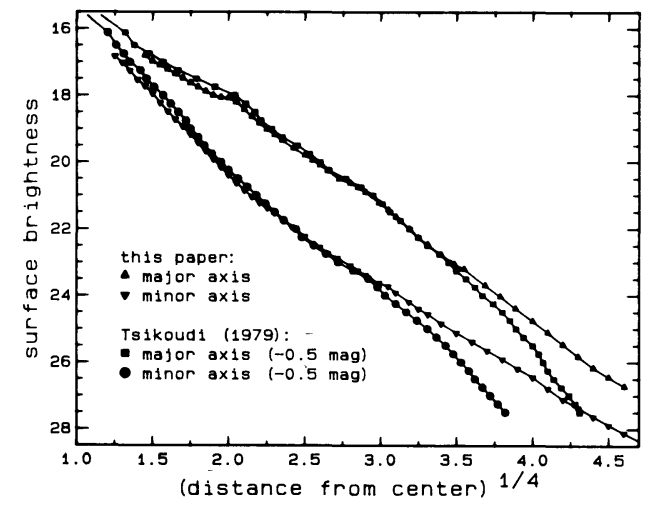

Figure 5. Main axis light profiles of NGC 3115 from Tsikoudi $(1978,1979)$ and from Capaccioli et al. (1986a). The graphical comparison discloses a large systematic discrepancy between the two sources, starting at $\mu \simeq 24 B-s s$.

If the list of known discrepancies is long, that of unknown mistakes is probably longer. Therefore it seems wise not to accept any photometric result on a single galaxy, which has not been satisfactory tested against a standard, or at least confirmed by independent investigations. Errors on individual cases may still remain, due to the peculiar role played by the background.

\section{LIGHT PROFILES AND FITTING LAWS}

de Vaucouleurs (1948) found that the light profiles of elliptical galaxies are approximately linear when the surface brightness is given as a function of the $1 / 4$ power of the radial distance from the center: $\log \left(I / I_{e}\right)=-3.3307\left[\left(r / r_{e}\right)^{1 / 4}-1\right]$. The so-called $r^{1 / 4}$ law, applied also to bulges of lenticulars and spirals, is now synonymous with elliptical galaxy. Not only observers are familiar with this law, but more and more theorists use it to build (e.g., Binney 1982b) or to test (e.g., van Albada 1982) their models. With respect to the other empirical formulae reviewed by dVC, the $r^{1 / 4}$ law has the advantage of giving a reasonable representation of the data, sometimes over a wide surface brightness range, with no need of free parameters. One modest drawback is that deprojection, and related mathematical operations, 
request cumbersome calculations (Young 1976); for this reason Jaffe (1983) has proposed another fitting law, which results from the projection of a simple analytic formula for the 3-D light distribution. Another problem is that the scale factor $r_{e}$ is ill defined observationally, since the quantity directly measured is $r_{e}^{1 / 4}$. The difficulty is often by-passed replacing $r_{e}$ with the half effective aperture, $r_{e}^{*}$, computed from growth curves. This parameter is not related to the fitting law and, from the methodological point of view, is more suitable to seek correlations with other observational quantities, e.g. with effective surface brightness (Kormendy 1980) or with absolute magnitude (Binggeli et al. 1984, Romanishin 1986).

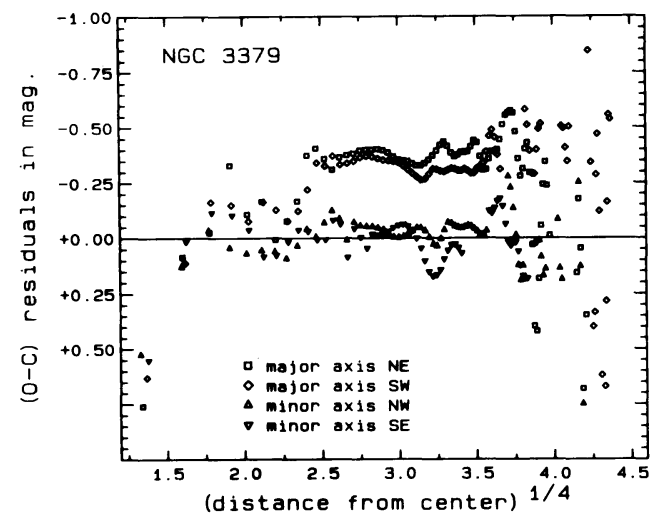

Figure 6. Magnitude differences between the main axis profiles of the standard galaxy NGC 3379 and the $r^{1 / 4}$ law best fitting the minor axis light distribution (Capaccioli et al. 1986b). The waves in the residuals repeat on both sides of each axis.

\subsection{Companions}

The choice of a parameter-free law to represent the entire light profiles of ellipticals, from the center to the outskirts, conceals the implicit assumption of the existence of a single characteristic photometric component. It is for this reason that we often speak of 'deviations' from the $r^{1 / 4}$ law as markers of additional components and of modifications induced in the radial distribution of light by the effect of a given environment. Major deviations occur in galaxy centers, where the actual profiles may be either steeper or shallower than the extrapolation of the $r^{1 / 4}$ law fitting of the outer parts (Schweizer 1979, dVC, Kormendy 1985). Other significant deviations occur in the faint tails of the light profiles, which are tidally truncated in dwarfs (King 1966, Binggeli et al. 1984) and exhibit an excess of light ('corona') over the extrapolation of the inner $r^{1 / 4}$ fit in cD's. This second effect, particularly strong in galaxies dominating dense clusters or groups (Thuan and Romanishin 1981, Sulentic and Lorre 1983, Schombert 1986), is often described by an exponentially truncated Hubble law (Oemler 1976), and is interpreted as the consequence of cannibalism in otherwise $r^{1 / 4}$ galaxies. Although less pronounced, a similar effect, pointed out by Kormendy (1977), occurs in the outer luminosity profiles of elliptical galaxies having close companions. The tidal stretching bends the profiles in such a way that the residuals from an unbiased $r^{1 / 4}$ fit over the entire range of measurements (except the galaxy core) have a characteristic parabolic trend. Actually this trend, recalling the behaviour of quasi-isothermal models (de Vaucouleurs 1953, King 1966), seems to be a characteristic of ellipticals, irrespective of the presence of close companion galaxies ( $c f$. Fig. 6 in Capaccioli 1984b). In conclusion, even in the most favourable cases (isolated objects), light profiles of ordinary ellipticals show real deviations up to $0.1-0.2 \mathrm{mag}$ from the $r^{1 / 4}$ law, which are possibly systematic and correlated with absolute luminosity (Michard 1985). 


\subsection{Ripples}

The E-W luminosity profile of the standard elliptical NGC 3379 shows a pattern of deviations from the $r^{1 / 4}$ behaviour which is different from those discussed above. The magnitude residuals from the smooth fitting law, plotted $v s$. distance from center, have a wavy trend with amplitude $\sim 0.1 \mathrm{mag}$ (Fig. 3 in dVC). The occurrence of such 'waves' has been confirmed by Nieto (1983a,b) and by Kent (1984), who has questioned their amplitude on the basis of CCD photometry in the red (dVC work is in the blue). Further information comes from Capaccioli et al. (1986b). From the reduction of two limiting Schmidt exposures they confirm, down to $\mu=28 B-s s$, the $\mathrm{E}-\mathrm{W}$ profile given by $\mathrm{dVC}$, and find that the waves in the residuals are symmetrically placed on opposite sides of the major and minor axis profiles (Fig. 6). So far no other bona fide elliptical has been discovered to show a similar behaviour, possibly because no other galaxy has been so extensively studied by the photometric technique as NGC 3379. Therefore speculations should wait for additional data. Nevertheless, in the following section we will attempt to explain the waves of the light profile of NGC 3379 by proposing that, as many other $\mathrm{E}$ galaxies, this object is a misclassified S0 seen almost face-on. If this is the case, the waves are simply structures in the disk.

\section{INTERLOPERS AMONG ELLIPTICAL GALAXIES}

Except for the faintest (Ichikawa et al. 1986) and possibly the brightest (Schombert 1986) end of the luminosity function of ellipticals, there is no clear evidence of discontinuity in the average photometric properties. This is true at least for those properties which are correlated with integrated parameters (e.g., ellipticity curves have no systematic trend for $e<0.4$; King 1978, Michard 1985). Still, remarkable differences occur between individual galaxies, which are partly accidental (occasional interactions) and partly intrinsic (Efstathiou and Fall 1984). An external contribution to the scatter may come from contamination of the pure $\mathrm{E}$ class by lenticulars. If, as suggested by Burstein (1978) and Simien and de Vaucouleurs (1986), the disk-to-bulge ratio in S0's can assume any value down to zero (a viewpoint which is not in conflict with the dichotomy between $\mathrm{E}$ and disk galaxies discussed by Bertola and Capaccioli, 1978), and if light profiles of bulges mimic those of ellipticals (a question which is still open; Kent 1985), then it is not surprising that some low $D / B$ lenticulars have been misclassified.

If it is a generalized phenomenon (e.g., see the very small number of E's in the sample of Corwin et al., 1985), misclassification may have some important astrophysical consequences. First, it alters the relative frequency of disk galaxies, which is a parameter relevant to our understanding of galaxy formation and evolution processes. Second, it may affect the statistics of intrinsic flattening, if the occurrence of interlopers is a function of their apparent flattening, and change the picture of intrinsic shapes, since bulges of lenticulars are oblate spheroids (Binney 1982a). Finally, it may obscure correlations among observational parameters (Capaccioli et al. 1984b). In principle, all cases of misclassified lenticulars should be easily identified by accurate photometric and kinematical observations when the faint disk is favourably inclined, i.e. when the objects are not far from edge-on. Flat lenticulars have a high rotational velocity (Capaccioli 1979), easy to measure. 
Their major axis light and color profiles differ from those along the minor axis; the latter have a shallower and smoother trend in luminosity (Fig. 3), and a steep color gradient (Strom et al. 1976). The same is true for structural features such as dust lanes and patches ( $c f$. Bertola, this volume), boxiness of the isophotes and their sharpening along the major axis (Capaccioli et al. 1986a).

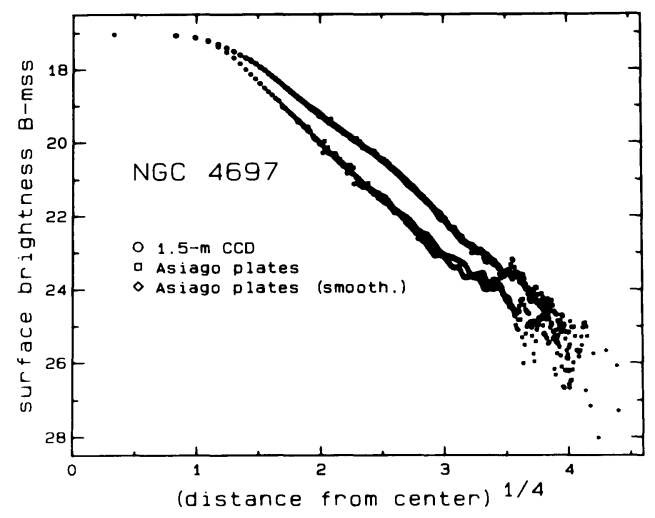

Figure 7. NGC 4697 exhibits the characteristic difference between the trends of major and minor axis light profiles, typical of lenticulars. Again the equivalent profile, not shown in the figure, is close to an $r^{1 / 4}$ law, as in King (1978).

The situation described above is precisely that of NGC 4125 and NGC 4697, classified ellipticals in the RC2 (de Vaucouleurs et al. 1976), and then discovered to be disk galaxies (Fig. 7). It is interesting to note that NGC 4697 is a fast rotator (Davies 1981); its rotation curve has the two relative maxima characteristic of lenticulars (Capaccioli 1979). A list of suspicious cases, such as NGC 4406, 4472 or 4649, may be compiled by comparing RC2 with RSA (Sandage and Tammann $1984)$ and with the original estimates by de Vaucouleurs (1963).

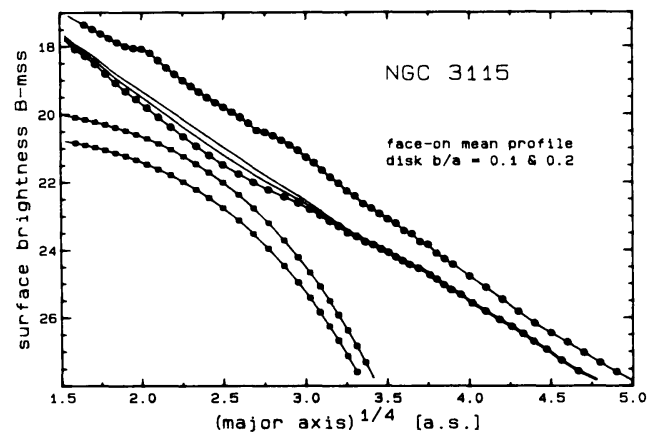

Figure 8. The full lines are face-on light profiles of NGC 3115 computed by adding the deprojected profiles of the bulge (lower dotted curve) and of the disk (triangles) for two values of the intrinsic flattening of the latter (Capaccioli et al. 1986a). The observed profile along the major axis (upper dotted curve) is also shown for comparison.

Let us now consider the problem of face-on lenticulars having a faint disk and not very prominent dust/absorption features; strong lanes as in NGC 404 (Sandage 1961) would immediately reveal the nature of the object. The question is: will the light distribution in such a galaxy mimic that of a pure elliptical ? A positive answer has been given by Capaccioli et al. (1986a). They were able to compute the 
face-on light profile of the lenticular NGC 3115, showing that, except for details in the disk, it follows very closely an $r^{1 / 4}$ law (Fig. 8), while the actual profiles along both axes do not (Fig. 5). In conclusion, unless peculiar features mark the image, or the lens is strong enough to produce the typical 'step' in the light distribution, an almost face-on bona fide lenticular can be easily confused with an elliptical galaxy. This is possibly the case for the E1 galaxy NGC 3379, which has a color gradient inconsistent with a spherical object (Strom et al. 1976) and a flattening curve similar to that of edge-on S0's (Fig. 9; cf. also Michard 1985). As we saw, the fact that, in the mean, its luminosity profiles follow well the $r^{1 / 4}$ law over a range of $8 \mathrm{mag}$, does not tell us much about its morphological type. Instead, the mysterious wavy residuals with respect to a smooth trend, mentioned above, would be fully understood if standard elliptical NGC 3379 were a misclassified lenticular.

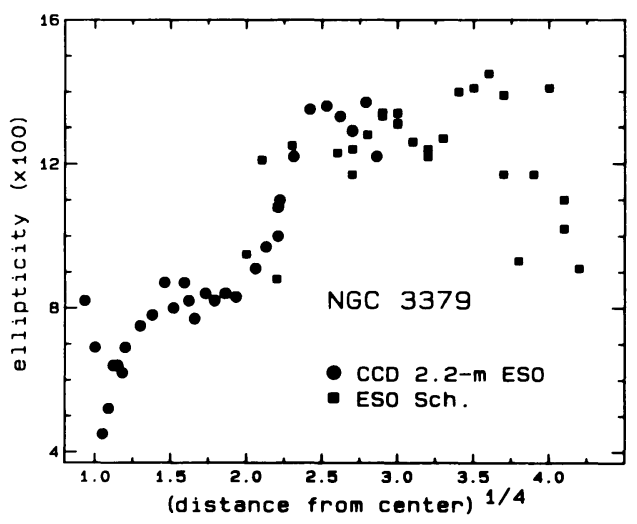

Figure 9. Ellipticity curve of NGC 3379 from Capaccioli et al. (1986b), obtained combining CCD and photographic data.

Part of the results presented here come from work done in collaboration with Prof. Gerard de Vaucouleurs and Dr.s Enrico Held, Jean-Luc Nieto and Roberto Rampazzo, to be published in full elsewhere. I would like to thank Dr. Jack W. Sulentic for comments and discussions.

\section{REFERENCES}

Barbon, R., Benacchio, L. and Capaccioli, M. 1976a. Mem. Soc. Astr. It., 47, 263.

Barbon, R., Benacchio, L. and Capaccioli, M. 1976b. Astron. Astrophys., 51, 25.

Barbon, R., Capaccioli, M. and Rampazzo 1982. Astron. Astrophys., 115, 388.

Bertola, F. and Capaccioli, M. 1975. Astrophys. J., 200, 439.

Bertola, F. and Capaccioli, M. 1978. Astrophys. J. Lett., 219, L95.

Bertola, F., Bettoni, D. and Capaccioli, M. 1982. In: Internal Kinematics and Dynamics of Galaxies, IAU Symposium No. 100, (Besançon), ed. E. O. Athanassoula, D. Reidel, p. 311.

Binggeli, B., Sandage, A. and Tarenghi, M. 1984. Astron. J., 89, 64.

Binney, J. 1982a. Ann. Rev. Astron. Astrophys., 20, 399.

Binney, J. 1982b. Mont. Not. R. astr. Soc., 200, 951.

Burstein, D. 1979. Astrophys. J. Suppl., 41, 435.

Capaccioli, M. 1979. In: Photometry, Kinematics and Dynamics of Galaxies, (Austin), ed. D. S. Evans, The Univ. of Texas Press, p. 165. 
Capaccioli, M. 1984a. In: Data Analysis in Astronomy, ed.s V. Di Gesu, L. Scarsi,

P. Crane, J. H. Friedman and S. Levialdi, (Erice), Plenum Press, p. 363.

Capaccioli, M. 1984b. In: New Aspects of Galaxy Photometry, (Toulouse), ed.

J.-L. Nieto, Springer-Verlag, p. 53.

Capaccioli, M. and de Vaucouleurs, G. 1983. Astrophys. J. Suppl., 52, 465 (CdV).

Capaccioli, M. and Rampazzo, R. 1986. preprint.

Capaccioli, M., Held, E. and Lorenz, H. 1986b. in preparation.

Capaccioli, M., Held, E. V. and Nieto, J.-L. 1984a. In: New Aspects of Galaxy

Photometry, (Toulouse), ed. J.-L. Nieto, Springer-Verlag, p. 265.

Capaccioli, M., Held, E. V. and Nieto, J.-L. 1986a. Astrophys. J. Suppl., submitted.

Capaccioli, M., Held, E. V. and Rampazzo, R. 1984b. Astron. Astrophys., 135, 89.

Capaccioli,M., Davoust, E., Lelievre, G. and Nieto, J.-L. 1983. In: Astronomy with Schmidt-type Telescopes, IAU Colloquium No. 78, (Asiago), ed. M. Capaccioli, D. Reidel, p. 379.

Carter, D. and Dixon, K. L. 1978. Astron. J., 83, 574.

Corwin, H. G., de Vaucouleurs, A. and de Vaucouleurs, G. 1985. Southern Galaxy Catalogue, The Univ. of Texas Monographs in Astronomy, Austin, No. 4.

Davies, R. L. 1981. Mont. Not. R. astr. Soc., 194, 879.

Davoust, E. and Pence, W. D. 1982. Astron. Astrophys. Suppl., 49, 631.

de Vaucouleurs, G. 1948. Ann. Astrophys., 11, 247.

de Vaucouleurs, G. 1953. Mont. Not. R. astr. Soc., 113, 134.

de Vaucouleurs, G. 1958. Astrophys. J., 128, 65.

de Vaucouleurs, G. 1959. Hand. der Phys., 53, 331.

de Vaucouleurs, G. 1963. Astrophys. J., 8, 31.

de Vaucouleurs, G. 1968. Appl. Opt., 7, 1513

de Vaucouleurs, G. 1979. In: Photometry, Kinematics and Dynamics of Galaxies, (Austin), ed. D. S. Evans, The Univ. of Texas Press, p. 1.

de Vaucouleurs, G. 1983. In: Astronomy with Schmidt-type Telescopes, IAU Colloquium No. 78, (Asiago), ed. M. Capaccioli, D. Reidel, p. 367.

de Vaucouleurs, G. and Capaccioli, M. 1979. Astrophys. J. Suppl., 40, 699 (dVC).

de Vaucouleurs, G. and Nieto, J.-L., 1978. Astrophys. J., 220, 449.

de Vaucouleurs, G. and Nieto, J.-L., 1979. Astrophys. J., 230, 697.

de Vaucouleurs, G., de Vaucouleurs, A. and Corwin, H. G. 1976. Second Reference

Catalogue of Bright Galaxies, Univ. of Texas Press, Austin (RC2).

Djorgowski, S. 1986. Ph. D. Thesis, Univ. of California at Berkeley.

Efstathiou, G. and Fall, M. S. 1984. Mont. Not. R. astr. Soc., 206, 453.

Fort, B. P., Prieur, J.-L., Carter, D., Meatheringam, S. J. and Vigroux, L. 1986. Astrophys. J., 306, 110.

Fraser, C. 1977. Astron. Astrophys. Suppl., 29, 161.

Hubble, E. 1930. Astrophys. J., 71, 231.

Ichikawa, S.-I., Wakamatsu, K.-I. and Okamura, S. 1986. Astrophys. J. Suppl., 60, 475.

Illingworth, G. 1977. Astrophys. J. Lett., 218, L43.

Jaffe, W. 1983. Mont. Not. R. astr. Soc., 202, 995.

Jones, W. B., Obbits, D. L., Gallet, R. M. and de Vaucouleurs, G. 1967. Publ. Dept. Astron., Univ. of Texas, Austin, Ser. 2, 1, No. 8.

Kent, S. 1984. Astrophys. J. Suppl., 56, 105.

Kent, S. 1985. Astrophys. J. Suppl., 59, 115. 
King, I. R. 1966. Astron. J., 71, 64.

King, I. R. 1978. Astrophys. J., 222, 1.

Kormendy, J. 1977. Astrophys. J., 218, 333.

Kormendy, J. 1980. In: Proc. ESO Workshop on Two-dimensional Photometry, ed.s P. Crane and K. Kjar, (Leiden: Sterrewacht), p. 191.

Kormendy, J. 1982. In: Morphology and Dynamics of Galaxies, XII Advanced Course of Swiss Soc. Astron. Astrophys., ed.s L. Martinet and M. Major, (Sauverny: Geneva Obs.), p. 115.

Kormendy, J. 1985. Astrophys. J., 292, L9.

Lauberts, A. and Valentijn, E. 1984. In: New Aspects of Galaxy Photometry, (Toulouse), ed. J.-L. Nieto, Springer-Verlag, p. 72.

Lauer, T. R. 1985. Astrophys. J. Suppl., 57, 473.

Liller, M. 1960. Astrophys. J., 132, 306.

Liller, M. 1966. Astrophys. J., 146, 28.

Malin, D. F. and Carter, D. 1980. Nature, 285, 643.

Malumuth, E. M. and Kirshner, R. P. 1984. Astrophys. J., 291, 8.

Michard, R. 1985. Astron. Astrophys. Suppl., 59, 205.

Miller, R. H. 1963. Astrophys. J., 137, 733.

Miller, R. H. and Prendergast, K. H. 1968. Astrophys. J., 153, 35.

Nieto, J.-L. 1983a. Astron. Astrophys. Suppl., 53, 247.

Nieto, J.-L. 1983b. Astron. Astrophys. Suppl., 53, 383.

Oemler, A. 1976. Astrophys. J., 209, 693.

Pence, W. D. and Davoust, E. 1985. Astron. Astrophys. Suppl., 60, 517.

Piotto, G. 1986. Ph. D. Thesis, University of Padova.

Romanishin, W. 1986. Astron. J., 91, 76.

Sandage, A. 1961. The Hubble Atlas of Galaxies, Carnegie Inst. of Washington, Publ. No. 618.

Sandage, A. and Tammann, G. 1984. A Revised Shapley-Ames Catalogue, Carnegie Inst. of Washington, Publ. No. 635 (RSA).

Schweizer, F. 1979. Astrophys. J., 233, 23.

Schombert, J. M. 1986. Astrophys. J. Suppl., 60, 603.

Simien, F. and de Vaucouleurs, G. 1986. Astrophys. J., 302, 564.

Strom, S. E., Strom, K. M., Goad, J. W., Vrba, F. J. and Rice, W. 1976. Astrophys. J., $204,684$.

Stetson, P. B. 1983. DAOPHOT User's Manual.

Sulentic, J. W. and Lorre, J. J. 1983. Astron. Astrophys., 120, 36.

Thuan, T. X. and Romanishin, W. 1981. Astrophys. J., 248, 439.

Tsikoudi, V. 1978. Ph.D Thesis, The Univ. of Texas, Austin.

Tsikoudi, V. 1979. Astrophys. J., 234, 842.

van Albada, D. 1982. Mont. Not. R. ast. Soc., 201, 939.

Watanabe, M. 1983. Ann. Tokyo Astrophys. Obs., 2nd Ser., 19, 121.

Williams, T. B. and Schwarzschild, M. 1979. Astrophys. J., 227, 56.

Young, P. J. 1976. Astron. J., 81, 807.

\section{DISCUSSION}

Djorgovski: Some ways of plotting the data, e.g., $\log I$ vs. $r^{1 / 4}$, stretch the profiles so that almost anything looks linear. The picture is visually dominated by the strong radial gradient. I think that the only fair and honest way of comparing data to fitting functions and models is to plot the residuals. 
Capaccioli: I fully agree.

Burstein: In the on-going investigation of elliptical galaxies by the "Gang of Seven", we compared CCD surface photometry with photoelectric photometry for about 30 galaxies. As far as we can determine, we have as much trouble with different zero points among the photo-electric photometry, as among the CCD data. In particular, we compared CCD four-shooter data on NGC 4697 to $\sim 15$ photoelectric aperture measures. The mean deviation, (CCD-P.E.) was $\pm 0.02 \mathrm{mag}$, i.e., we have not had serious problems in connecting photo-electric and CCD data.

Capaccioli: Very much reassuring! However, I think that your nice results depend also on the fact that you have new photo-electric data. My remark is relative to old published photo-electric data.

Porter: CCD's are potentially more helpful than you imply if they can be used in drift scan mode, being read out continuously as the telescope tracks in the opposite direction. A whole new set of calibration problems arise (sky brightness should be monitored independently, color terms may be different, and the telescope's tracking must be excellent), but you can take strip images long enough to reach "true sky". Those images are very flat, and would allow direct comparison of electronic and photographic photometry over the galaxy's entire range of surface brightness.

Capaccioli: All that is very true. Yet I think it is more economical to combine CCD's with photographic photometry.

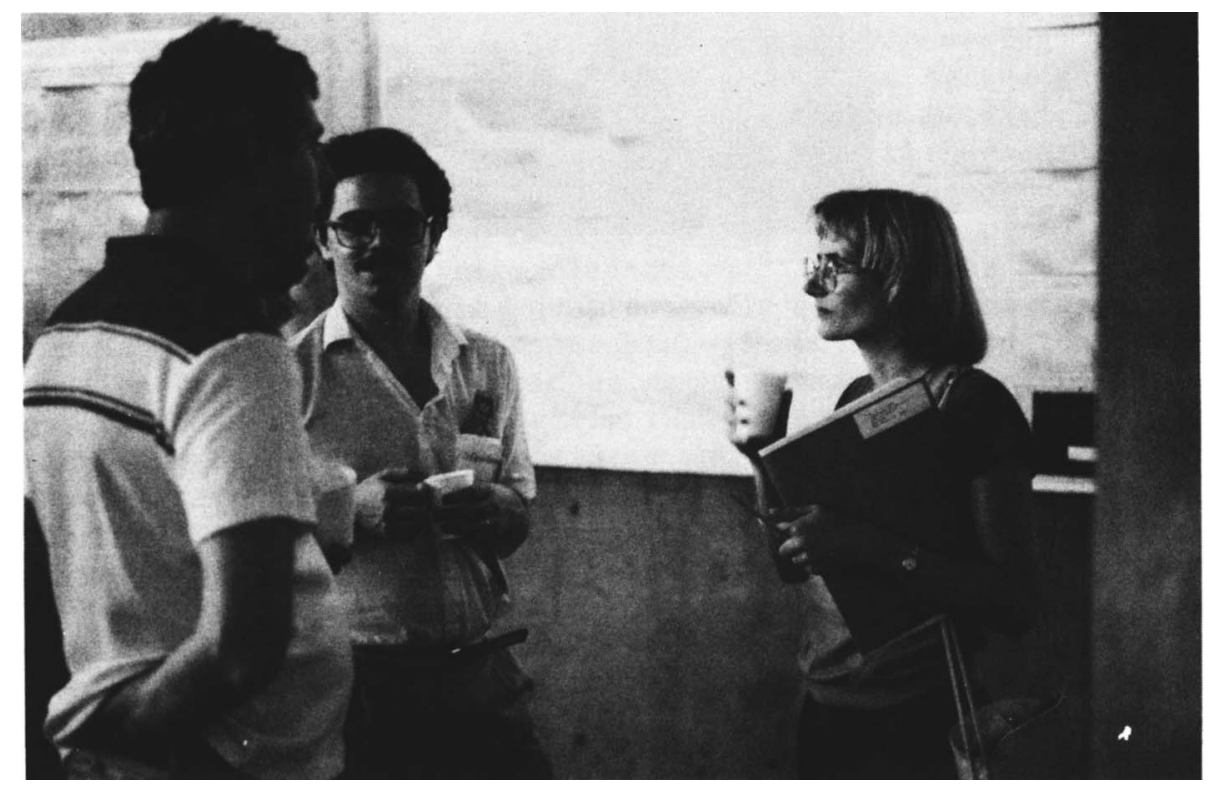

Stefano Casertano, chairman of the LOC, has a rare moment of rest with Andrew Pickles and Patricia Vader. 


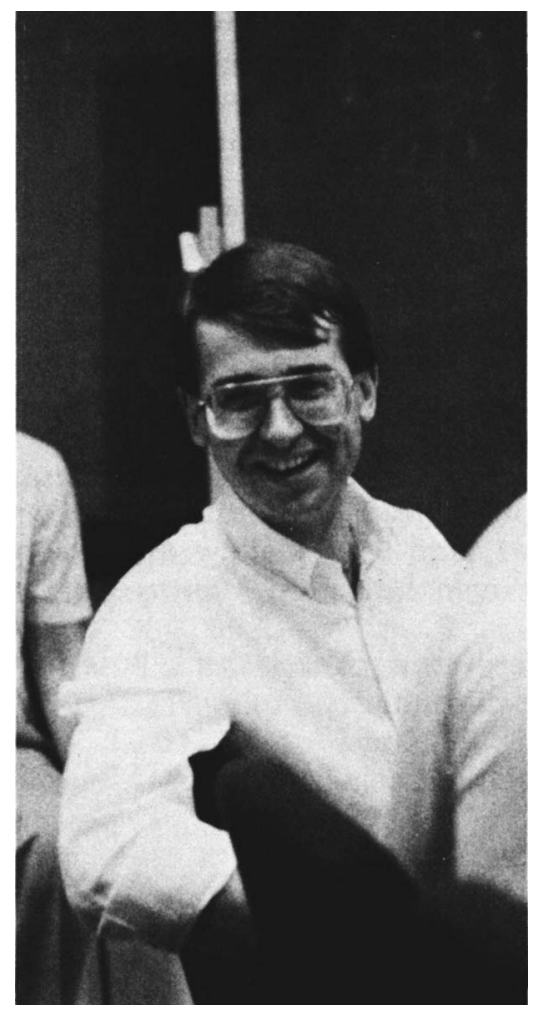

Roger Davies enjoying himself. 